\author{
Simos Evangelou \\ Department of Electrical and Electronic \\ Engineering, \\ and Department of Mechanical Engineering, \\ Imperial College London, \\ London SW7 2AZ, UK \\ e-mail: s.evangelou@imperial.ac.uk
}

\author{
David J. N. Limebeer ${ }^{1}$ \\ e-mail: d.limebeer@imperial.ac.uk
}

\section{Maria Tomas Rodriguez \\ e-mail: maria.tomas@imperial.ac.uk}

Department of Electrical and Electronic

Engineering,

Imperial College London,

London SW7 2AZ, U.K

\title{
Influence of Road Camber on Motorcycle Stability
}

This paper studies the influence of road camber on the stability of single-track road vehicles. Road camber changes the magnitude and direction of the tire force and moment vectors relative to the wheels, as well as the combined-force limit one might obtain from the road tires. Camber-induced changes in the tire force and moment systems have knock-on consequences for the vehicle's stability. The study makes use of computer simulations that exploit a high-fidelity motorcycle model whose parameter set is based on a Suzuki GSX-R1000 sports machine. In order to study camber-induced stability trends for a range of machine speeds and roll angles, we study the machine dynamics as the vehicle travels over the surface of a right circular cone. Conical road surfaces allow the machine to operate at a constant steady-state speed, a constant roll angle, and a constant road camber angle. The local road-tire contact behavior is analyzed by approximating the cone surface by moving tangent planes located under the road wheels. There is novelty in the way in which adaptive controllers are used to center the vehicle's trajectory on a cone, which has its apex at the origin of the inertial reference frame. The results show that at low speed both the weave-and wobble-mode stabilities are at a maximum when the machine is perpendicular to the road surface. This trend is reversed at high speed, since the weave- and wobble-mode dampings are minimized by running conditions in which the wheels are orthogonal to the road. As a result, positive camber, which is often introduced by road builders to aid drainage and enhance the friction limit of fourwheeled vehicle tires, might be detrimental to the stability of two-wheeled machines. [DOI: 10.1115/1.2937140]

\section{Introduction}

The role of road camber on the stability of motorcycles is an issue that arises in the context of loss-of-control accident investigations when high-speed cornering is involved. Superelevated roads are commonplace, and their banking is designed primarily to assist drainage and to improve the tire adhesion limit of fourwheeled vehicles. In the context of training, advanced police motorcycle riders are taught about the influence of road camber on tire adhesion using simple static-equilibrium-based ideas [1]. They are taught that superevaluated cross fall has a favorable influence on the vehicle's behavior, because it makes the road wheels more nearly vertical relative to the road. The teaching in Ref. [1] does not extend to the role of camber on machine stability, because this is a considerably more complex issue.

Our aim is to investigate the influence of road camber on the primary oscillatory modes of motorcycles. Insight is obtained from a high-fidelity computer model that has been developed for this purpose. In "flat-road" models the tires' normal load is parallel with the earth's gravitational field, while the tires' lateral and longitudinal forces are orthogonal to it. Similarly, the tires' aligning moment is in the direction of gravity, while the overturning and rolling resistance moments lie in a horizontal ground plane. In the case of cambered roads, the tires' normal load is no longer parallel to gravity, and the roadway is no longer horizontal or flat. The model presented here studies the consequences of these changes in detail.

The earliest dynamic models for two-wheeled vehicles go back over a century and were developed in the context of the bicycle. In the same way, early research on motorcycle dynamics was confined to small perturbations from straight running and relatively simple vehicle models. References $[2,3]$ provide a comprehensive

\footnotetext{
${ }^{1}$ Corresponding author.

Contributed by the Applied Mechanics Division of ASME for publication in the JouRnal of APPLIED Mechanics. Manuscript received July 18, 2007; final manuscript received January 7, 2008; published online August 21, 2008. Review conducted by N. Sri Namachchivaya.
}

review of much of this early literature. Simple models with rigidbody type frame representations, and constraint-based tire representations predict a single oscillatory mode known as "weave." Following the introduction of frame flexibility, and/or force generating tire models, a second oscillatory mode is predicted, which is known as "wobble." In the case that one or more of these modes is stable, but lightly damped, the potential exists for undesirable vehicle behavior. In later work, these models were extended to include small perturbations from a steady-state cornering condition [4-9]. It is clear from these studies that under certain operating conditions some of the machine's modes can be lightly damped, or even unstable. This theoretical work has been supplemented by extensive measurement programs [10-24].

The weave mode combines rolling, yawing, and steering in a fish-tailing motion and is well damped at moderate speeds, but becomes less so as the machine's forward speed increases. The natural frequency of this mode rises from zero at very low speed to somewhere in the range of $2-4 \mathrm{~Hz}$, depending on the mass and size of the machine; the lower frequencies correspond to heavier motorcycles. The wobble mode involves primarily a caster shimmy type steering oscillation, and has an oscillatory frequency that is essentially independent of speed and is normally in the $6-9 \mathrm{~Hz}$ range, the precise frequency being governed primarily by the mechanical trail, the front tire cornering stiffness, and the front frame steer inertia [25]. Computer simulation studies indicate that the torsional stiffness of the motorcycle frame at the steering head determines whether a machine will be prone to wobbling at medium speeds (a compliant frame) or at high speeds (a stiff frame) $[26,27]$. In cornering, the lateral (out-of-plane) modes and the in-plane modes associated with tire deflections and suspension motions become coupled, as was first shown in any detail by Koenen [4]. The motorcycle becomes prone to resonant forcing, which is induced by regular road undulations, when the displacement forcing they produce is tuned to the lightly damped modes of the machine. Moderate roll angles and high speeds are likely to represent the worst case conditions [28].

This paper is organized as follows. Section 2 describes the 


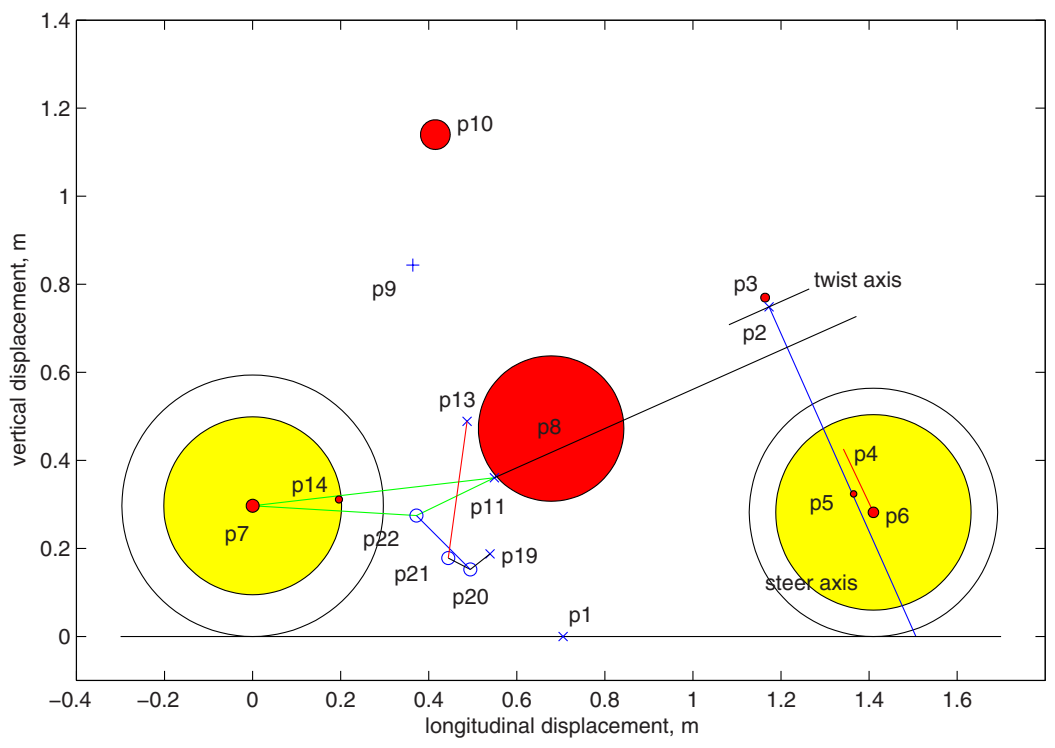

Fig. 1 Scaled diagrammatic side-view of the motorcycle model in its nominal configuration. The seven constituent bodies are shown as (dark) circles, with their radii proportional to their mass. All the points critical to building the model are individually marked. For example, the largest mass is the rear frame with its mass center located at p8.

background to the motorcycle model that will be used in this study. Some of the important characteristics of the standard priorart motorcycle-rider system are described in Sec. 2.1. The modeling of cambered roads is described in Sec. 2.2, where the computation of road normals and tangent planes is described. The standard motorcycle model control systems are extended in Sec. 2.3, where a new adaptive roll-angle control system is introduced. A single-wheel model that is used to study roll-angle equilibria and tire adhesion limits is introduced in Sec. 2.4 and analyzed in Sec. 2.5. This model is based on a "thick tire" and is used to study force and moment balances as well as certain aspects of the roadtire contact geometry. The machine's static stability limits are studied in Sec. 2.6. The influence of road camber on a highfidelity motorcycle model is analyzed in Sec. 3 with the conclusions drawn in Sec. 4.

\section{Motorcycle Model}

2.1 Prior Model. The motorcycle model used in this study is representative of modern road-going sports machines, and has a parameter set that is based on the Suzuki GSX-R1000. The machine geometry is shown in Fig. 1, in which the motorcycle's seven constituent masses are represented by (dark) circles that have a diameter that is proportional to the mass of the associated body. All the critical points (mass centers, linkage pivot points, and so on) are individually marked. The main frame is allowed unrestricted translational and rotational motions. The swinging arm, the monoshock linkage system, the rider, and front frame are all pinned to it. The (rear) monoshock suspension is restrained by a parallel spring and damper, while the front suspension is based on a standard telescopic fork that allows linear in-line displacements. The main frame is modeled as a single rigid body save for a torsional frame flexibility degree of freedom at the steering head-this freedom accommodates small angular displacements that are perpendicular to the steering axis. Aerodynamic influences are modeled using drag and lift forces, which are proportional to the square of the speed. The road tires are treated as "wide," flexible in compression, and care is taken to track dynamically the migration of both ground-contact points as the machine rolls, pitches, and steers. Both contact points, which are taken as the points closest to the road surface, will move laterally and circumferentially over the tires' surface; these points represent the center of the road-tire contact patch. The tire forces and moments are generated from the normal load, the tires' camber angle relative to the road, and the combined slip using "magic formalas" [29-32]. The lateral compliance of the tires' carcass is modeled using standard linear time-varying relaxation length type tire models [31]. Relaxation effects have a lagging influence on the generation of the lateral forces and aligning moments.

The machine's trim state is controlled using feedback loops, which control the steering torque and the drive to the rear wheel. The drive moment is controlled using a proportional-integral controller that acts on a speed error; this allows the vehicle to follow an arbitrary speed reference. The steering controller is proportional integral derivative in its general structure, and acts on a roll-angle error. The steering controller allows the vehicle to maintain a fixed roll angle, or follow a low-bandwidth roll-angle reference signal. A motorcycle model incorporating all of the above features has its origins in Ref. [5], and is extended in Refs. $[8,9]$. The model is written in LISP and makes use of the multibody modeling code AUTOSIM [33] and can be obtained from the website. ${ }^{2}$ The Autosim model can be configured to solve the nonlinear equations of motion, or it can be used to generate a symbolic state-space representation that describes small perturbation around a prescribed trim condition. For the purposes of this study, both the tire and controller modeling had to be extended. These extensions are described next.

2.2 Cambered Road Modeling. Suppose that the road over which the vehicle will travel is described in Cartesian coordinates by the differentiable road-surface function

$$
S(x, y, z)=0
$$

A general point $P$ on the road surface $S$ is denoted $S(P)$, and is described by the vector

\footnotetext{
${ }^{2} \mathrm{http} / / / \mathrm{www}$. imperial.ac.uk/controlandpower/motorcycles/
} 


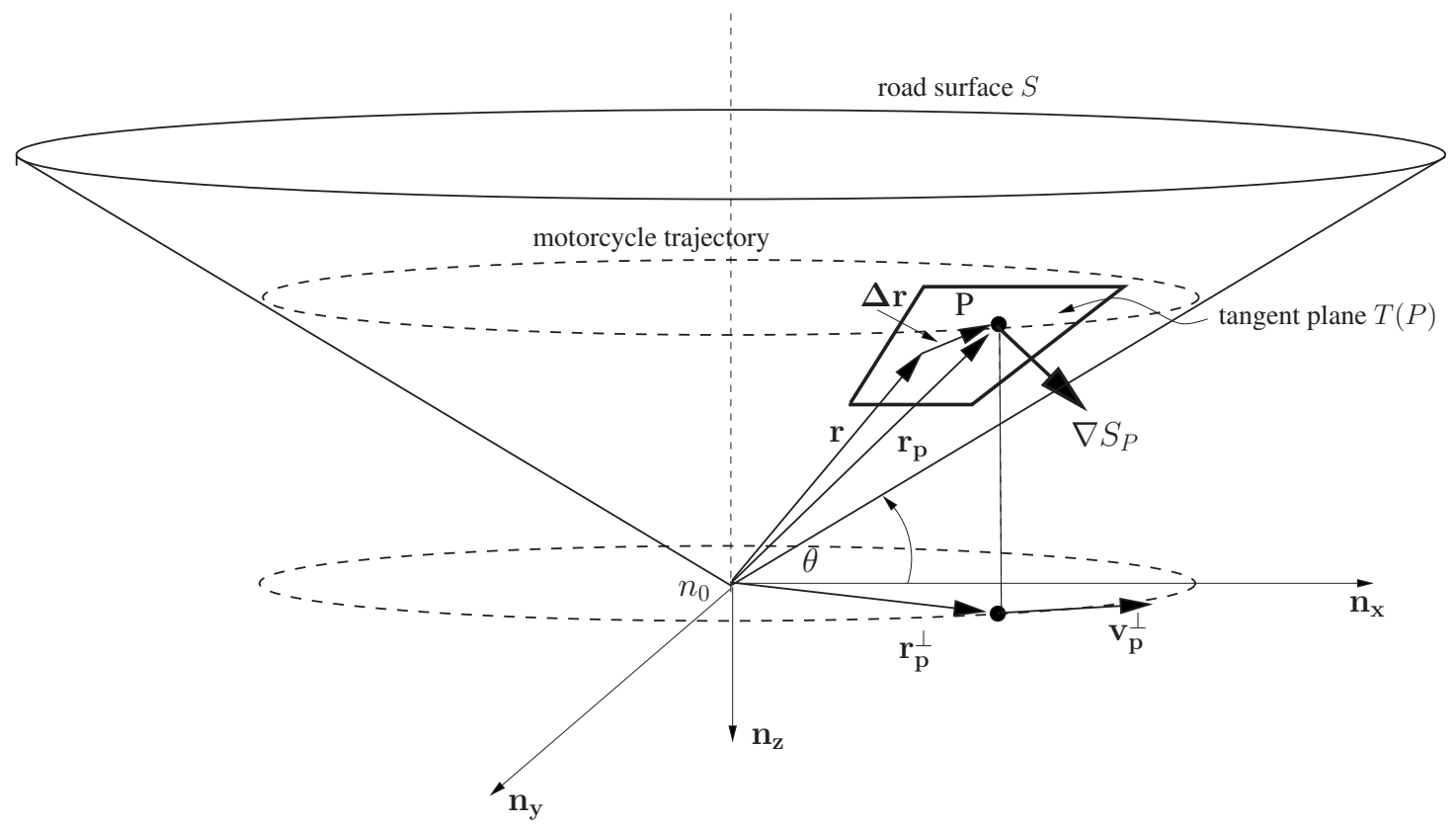

Fig. 2 The road surface used for cambered road stability studies is a right circular cone (as illustrated, the cone is inverted for positive camber angles in the range $90 \mathrm{deg}>\theta \geqslant 0$ ). The central axis of the cone is aligned with the inertial axis $n_{z}$, with its vertex at the origin $n_{0}$ of the inertial reference system. For camber angles in the range 90 deg $>\theta \geqslant 0$, the motorcycle is assumed to ride on the interior surface of the cone along a circular trajectory. Only positive yaw rate operating conditions are considered (clockwise when seen from above), which means that for positive roll angles the machine leans toward the central axis of the cone. The nominal rear-wheel ground-contact point is $P$. The actual rear-wheel ground-contact point is assumed to move over the tangent plane $T(P)$; the normal to the plane $T(P)$ is the vector $\nabla S_{P .}$ A second tangent plane is used to describe locally the road surface under the front wheel. The vector $r_{p}^{\perp}$ is the projection of $r_{p}$ onto the ground plane, while $\mathrm{v}_{p}^{\perp}$ is the velocity of $P$ projected onto the ground plane.

$$
\mathbf{r}_{P}=x_{P} \mathbf{n}_{x}+y_{P} \mathbf{n}_{y}+z_{p} \mathbf{n}_{z}
$$

in which $\mathbf{n}_{x}, \mathbf{n}_{y}$, and $\mathbf{n}_{z}$ are the standard basis vectors in a SAE coordinate system. A normal to the surface at $S(P)$ is given by ([34], p. 285)

$$
\nabla S_{P}=\left.\frac{\partial S}{\partial x}\right|_{P} \mathbf{n}_{x}+\left.\frac{\partial S}{\partial y}\right|_{P} \mathbf{n}_{y}+\left.\frac{\partial S}{\partial z}\right|_{P} \mathbf{n}_{z}
$$

A tangent plane $T(P)$, which contains the point $S(P)$, is described by

$$
\left\langle\left(\mathbf{r}-\mathbf{r}_{P}\right), \nabla S_{P}\right\rangle=0
$$

for any vector $\mathbf{r}-\mathbf{r}_{P}$ in $T(P)$. This follows from the fact that $\mathbf{r}$ $-\mathbf{r}_{P}$ must be perpendicular to any normal to the plane.

In the context of the present study, we are particularly interested in conical road surfaces, because they allow motorcycles to operate at trim conditions that are characterized by a prescribed constant speed, a constant roll angle, and a constant road camber angle. Figure 2 illustrates such a conical road surface and is described by

$$
S(x, y, z)=\sqrt{x^{2}+y^{2}} \tan \theta+z=0
$$

A unit-length normal at $S(P)$ is given by

$$
\nabla S_{P}=\left.\frac{x \sin \theta}{\sqrt{x^{2}+y^{2}}}\right|_{P} \mathbf{n}_{x}+\left.\frac{y \sin \theta}{\sqrt{x^{2}+y^{2}}}\right|_{P} \mathbf{n}_{y}+\cos \theta \mathbf{n}_{z}
$$

If $\mathbf{r}$ defines a general point in $T(P)$, the vector $\Delta \mathbf{r}=\mathbf{r}-\mathbf{r}_{P}$ is orthogonal to $\nabla S_{P}$ if it lies in $T(P)$. Thus, the tangent plane $T(P)$ in Fig. 2 is defined by

$$
\left(x-x_{P}\right) \frac{x_{P} \sin \theta}{\sqrt{x_{P}^{2}+y_{P}^{2}}}+\left(y-y_{P}\right) \frac{y_{P} \sin \theta}{\sqrt{x_{P}^{2}+y_{P}^{2}}}+\left(z-z_{P}\right) \cos \theta=0
$$

for a general point $(x, y, z) \in T(P)$.

With these ideas in mind, the motorcycle model described in Sec. 2.1 was modified to accommodate conical road surfaces. The essential idea is to represent the road surface locally using tangent planes that move under each of the road wheels. These planes are characterized by normals computed at the nominal (associated with the nominal configuration) ground-contact points. These points are treated as fixed in the main frame's body-fixed coordinate system. Contact point excursions are then represented as motions over the two tangent planes, which are fully characterized by normal vectors of the type given in Eq. (6). In the case of flat-road models, these normal vectors are given by the static vector $\mathbf{n}_{z}$. For the purposes of tire force and moment calculations, it is necessary to compute normal loads and aligning moments in the direction of Eq. (6). The tire camber angles are calculated relative to the planes (7), rather than the horizontal ground plane. The lateral and longitudinal tire forces, as well as the overturning moment act in the plane described by Eq. (7) rather than the ground plane. These changes are straightforward to implement by reworking the tire kinematics calculations described previously in Ref. [9], for example.

These ideas are further illustrated in Fig. 3, which show part of a motorcycle road tire moving over an inclined road surface with camber angle $\theta$. As before, the motorcycle's roll angle $(\phi)$ is defined relative to the inertial $\mathbf{n}_{z}$-axis. Under these conditions, the tire camber angle is approximately $\phi-\theta$, and it is this angle that informs the magic formulas of the tires' camber angle status, and it is this angle that generates the tires' camber force. The normal load is perpendicular to the road, and the lateral force lies in the road surface.

2.3 Adaptive Steering Controller. Another challenge facing 


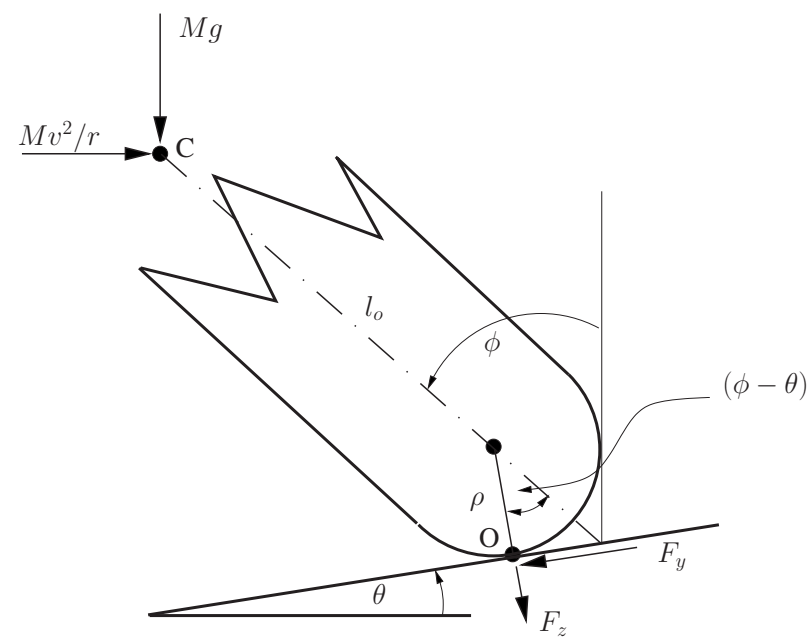

Fig. 3 Contact between an inclined road surface and a motorcycle tire in a single-wheel model. The total mass of the machine and rider is $M$, the total weight is therefore $M g$, and the centripetal force is $M v^{2} / r$. The tire crown radius is $\rho$, and the distance between the motorcycle's mass center $C$ and the center of the tire crown is $I_{o}$. The road camber angle is $\theta$, while the motorcycle roll angle is $\phi$; the motorcycle comes out of the page for positive yaw rates $(\dot{\psi}>0)$. The tire's normal load and side force are given by $F_{z}$ and $F_{y}$, respectively, and are applied at the contact point $O$. The force $F_{z}$ is normal to the road surface, while $F_{y}$ is tangent to it.

the motorcycle modeler, in the context of cambered road studies, is the correct positioning of the machine relative to the cone. When seeking to achieve a fixed-speed, fixed-roll-angle, and fixed-camber-angle trim condition, the first objective is to achieve the desired speed and roll-angle conditions. This can be done using known methods, but the center of the circular trajectory will be at an arbitrary location in the ground plane. The new aspect to the vehicle control problem is to move the fixed-speed fixed-rollangle trajectory toward the $\mathbf{n}_{z}$ axis so that it eventually rotates around it. This problem was solved using the adaptive-reference roll-angle feedback controller illustrated in Fig. 4. This scheme is informed by the fact that for a steady-state circular trajectory, the radial position vector and the corresponding velocity vector are orthogonal to each other. Intuitively, if an origin-centered trim trajectory is to be achieved, one should steer toward the origin if the angle between the position vector and the velocity vector is greater than $90 \mathrm{deg}$, and away from it if the angle is less than

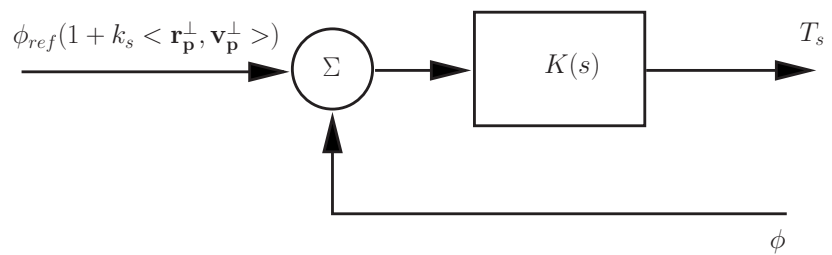

Fig. 4 Roll-angle feedback loop used in the simulation model. The steering torque $T_{s}$ is generated from the difference between the roll angle $\phi$ and the adaptive roll angle reference $\phi_{\mathrm{ref}}\left(1+\boldsymbol{k}_{s}\left\langle\mathrm{r}_{p}^{\perp}, \mathrm{v}_{p}^{\perp}\right\rangle\right) ; \mathrm{r}_{p}^{\perp}$ and $\mathrm{v}_{p}^{\perp}$ are defined in Fig. 2. If the motorcycle is moving toward the inertial axis $\mathrm{n}_{z}$, the adaptive gain term $k_{s}\left\langle r_{p}^{\perp}, v_{p}^{\perp}\right\rangle$ adjusts the roll-angle reference so as to steer the machine away from it. Conversely, if the motorcycle is moving away from $\mathrm{n}_{z}$, the adaptive gain term $\boldsymbol{k}_{s}\left\langle\mathrm{r}_{p}^{\perp}, \mathrm{v}_{p}^{\perp}\right\rangle$ steers the machine toward it. The adaptive roll-angle term thus has the effect of centering the machine trajectory on $n_{z}$ and becomes noncontributory once the machine's trajectory has been centered; in this event, $\left\langle\mathrm{r}_{p}^{\perp}, \mathrm{v}_{p}^{\perp}\right\rangle=0$.
$90 \mathrm{deg}$. If these vectors remain orthogonal, no position-related steering is required. These ideas are implemented in the adaptive control system illustrated in Fig. 4, which "adapts" the roll-angle reference to the correction term $k_{s}\left\langle\mathbf{r}_{p}^{\perp}, \mathbf{v}_{p}^{\perp}\right\rangle$. The gain $k_{s}$ is constant, and $\langle\cdot, \cdot\rangle$ represents the inner product between the machine's position and velocity vectors projected onto the ground plane. Clearly, as the angle between $\mathbf{r}_{p}^{\perp}$ and $\mathbf{v}_{p}^{\perp}$ passes through $90 \mathrm{deg}$, the sign of the inner product term changes. The stability of the system is obviously crucial, but a formal stability analysis appears prohibitively difficult to carry out. It turned out that this loop is easy to tune by trial and very effective in terms of its operation. Figure $5(a)$ shows the machine being moved by the adaptive reference scheme from an arbitrary ground-plane position to an origin-centered orbital trajectory. Figure 5(b) shows the machine operating under full feedback control as it slowly accelerates up the conical road surface. The roll angle is maintained at a constant reference value of $\phi=0 \mathrm{deg}$, while its speed is increased at a constant acceleration of $0.005 \mathrm{~m} / \mathrm{s}^{2}$.

2.4 Single-Wheel Model. Cambered road surfaces in combination with profiled tires make the prediction of viable operating conditions a nontrivial exercise. In order to establish if a particular operating condition represents a potentially stable equilibrium, we will study the conditions required for a roll-angle equilibrium as well as the possible violation of the road-tire adhesion limits. To do this, we will make use of the simple single-wheel model illustrated in Fig. 3, in which both road camber and tire profiling are taken into account. This single-wheel model is reminiscent of the inverted-pendulum type model described in Ref. [35], but with the steering locked in the straight-running position. In the context of this model, the important parameters are the tire profile and friction limit, the location of the machine's mass center, the road camber angle, and the machine's roll angle and speed. The distance $l_{o}+\rho$ is the height of the machine's mass center above a level road surface when it is in its nominal configuration. The parameter $\rho$ is the single-wheel tire crown radius and must be chosen to represent both wheels. It should be emphasized that this simple model can only be used for a restricted range of purposes, and when such things as the wheel's gyroscopic moments are justifiably neglected. Care has been taken to cross check the utility of this model with the high-fidelity model described in Sec. 2.1. In order to compare the single-wheel abstraction with the highfidelity model, representative values for $l_{o}$ and $\rho$ are required. For present purposes, we have set $\rho$ equal to the average value of the front and rear tire crown radii: $\rho=(0.095+0.06) / 2=0.0775 \mathrm{~m}$. One can then find a value for $l_{o}$ from the height of the machine's mass center. In its nominal configuration, the height of the mass center of the whole machine above the ground is $l=0.5091 \mathrm{~m}$, which gives $l_{o}=l-\rho=0.4316 \mathrm{~m}$. Necessary conditions for a stable steady-state roll equilibrium are studied next.

2.5 Equilibrium Modeling. Valuable insight into the influence of road camber on the steady-state tire force and moment system can be obtained from simple static-equilibrium based calculations. These calculations also provide insight into the machine's stable domain of operation, which is limited by its static roll stability and the tire friction limit. The machine has a stable trim state for only a limited range of negative roll-angle conditions; we will show that the precise conditions depend on the tire's crown radius and the tire friction limit. We begin by referring again to the single-wheel model illustrated in Fig. 3 and then resolve forces in the $F_{y}$ and $F_{z}$ directions, respectively. This gives

$$
F_{y}=M \frac{v^{2}}{r} \cos \theta-M g \sin \theta
$$



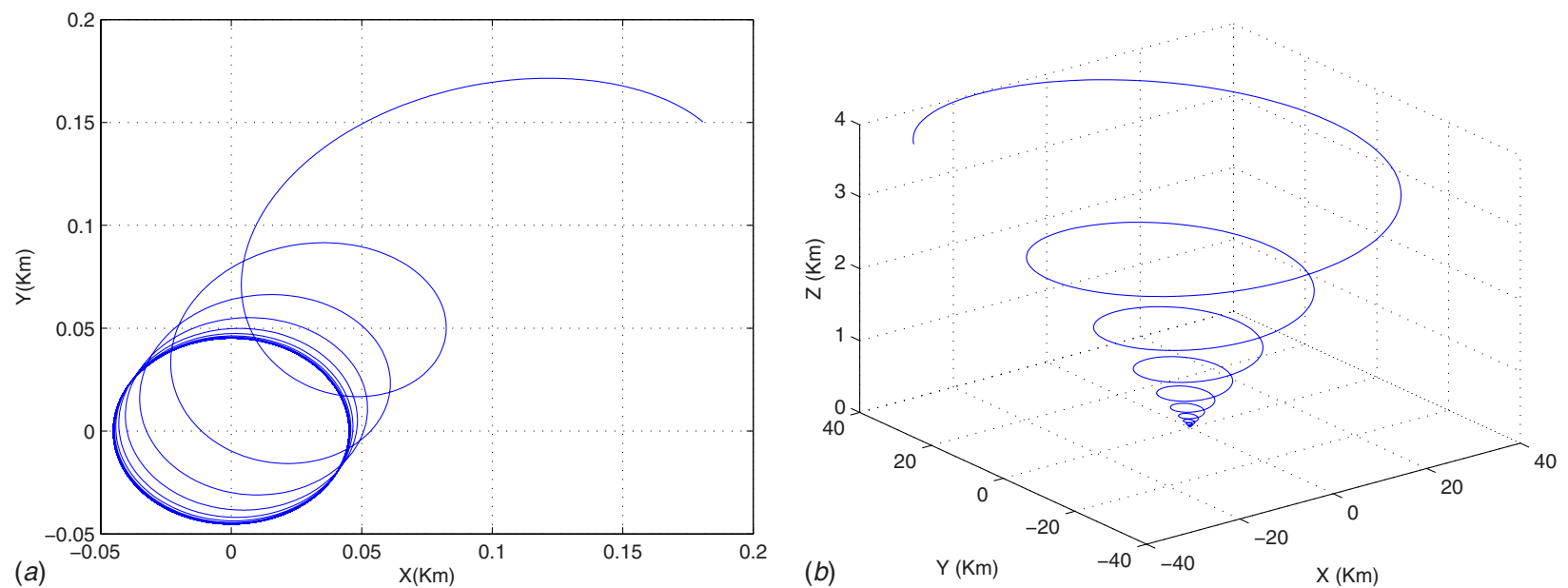

Fig. 5 Adaptive roll-reference controller centering the motorcycle trajectory on $\mathrm{n}_{z^{*}}$ (a) The machine trajectory begins at the initial point $(180,150)$ in the ground plane, which is outside the origin-centered equilibrium circle for the motorcycle cornering at $10 \mathrm{~m} / \mathrm{s}$ with a roll angle of $15 \mathrm{deg}$; under these conditions, the equilibrium radius of curvature is $45 \mathrm{~m}$. In this simulation, the adaptive roll-angle reference gain is $k_{s}=5.0 \times 10^{-4}$ (see Fig. 4). (b) Motorcycle running on the surface of a cone with camber angle $\theta=5 \mathrm{deg}$. In this simulation, the machine accelerates from $5 \mathrm{~m} / \mathrm{s}$ to $75 \mathrm{~m} / \mathrm{s}$ at $0.005 \mathrm{~m} / \mathrm{s}^{2}$, with the motorcycle roll angle maintained at $\phi=0$ deg; this trajectory begins at $(0,0)$ in the ground plane.

$$
F_{z}=-M \frac{v^{2}}{r} \sin \theta-M g \cos \theta
$$

Taking moments around the ground-contact point $O$ gives

$$
\frac{M v^{2}}{r}\left(l_{o} \cos \phi+\rho \cos \theta\right)=M g\left(l_{o} \sin \phi+\rho \sin \theta\right)
$$

from which it follows that machine trajectory has radius of curvature

$$
r=\frac{v^{2}\left(l_{o} \cos \phi+\rho \cos \theta\right)}{g\left(l_{o} \sin \phi+\rho \sin \theta\right)}
$$

under trim conditions. Eliminating $M v^{2} / r$ from Eqs. (8) and (9) using Eq. (11) gives

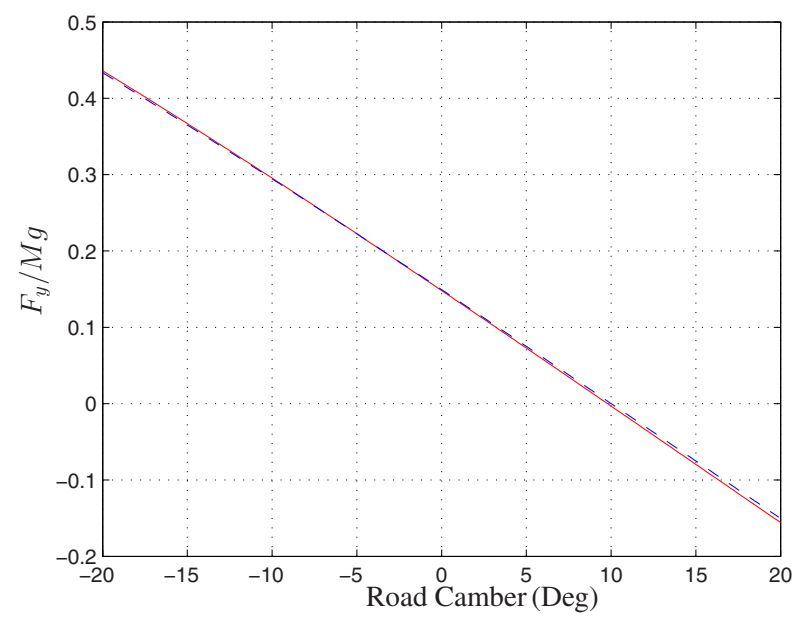

(a)

$$
F_{y}=\frac{M g l_{o} \sin (\phi-\theta)}{l_{o} \cos \phi+\rho \cos \theta}
$$

which simplifies to

$$
F_{y}^{\text {thin }}=\frac{M g \sin (\phi-\theta)}{\cos \phi}
$$

in the case of a "thin" tire $(\rho=0)$. In the same way,

$$
F_{z}=-\frac{M g\left(l_{o} \cos (\phi-\theta)+\rho\right)}{l_{o} \cos \phi+\rho \cos \theta}
$$

or

$$
F_{z}^{\text {thin }}=-\frac{M g \cos (\phi-\theta)}{\cos \phi}
$$

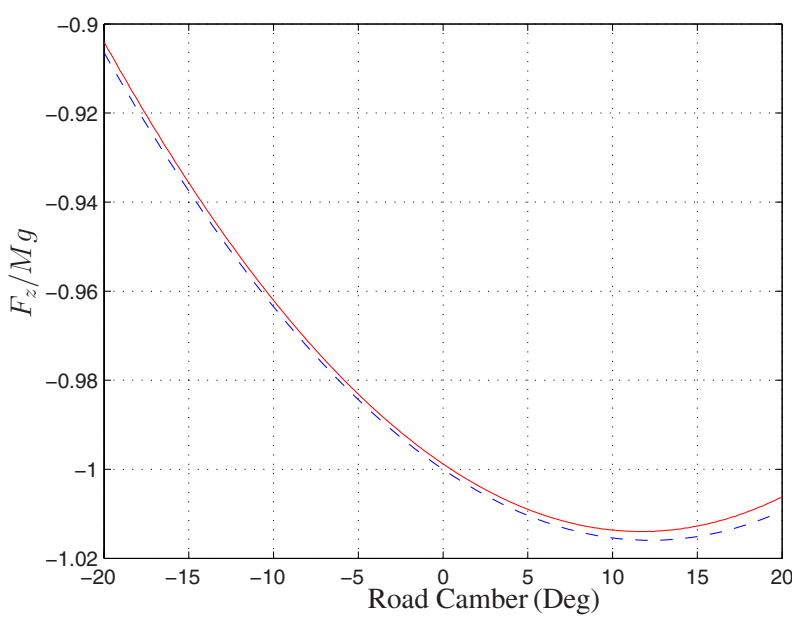

(b)

Fig. 6 Approximate and exact side force and normal loads as functions of camber angle; the machine roll angle is $\phi$ $=10$ deg. (a) Normalized side force; the dashed curve comes from Eq. (12), while the solid curve is computed using the high-fidelity model at a forward speed of $10 \mathrm{~m} / \mathrm{s}$. (b) Normalized normal load; the dashed curve comes from Eq. (13), while the solid curve is computed using the high-fidelity model. 
in the case of a thin tire.

Figure 6(a) shows the approximate normalized tire side-force calculated using Eq. (12) in comparison to the total normalized tire side force computed using the high-fidelity model described in Sec. 2.1. The agreement is very good, and as would be expected, $F_{y}$ vanishes when the motorcycle tire is perpendicular to the road. A similar comparison appears in Fig. 6(b), where the approximate and exact normal loads are compared as a function of camber angle. Again, as is expected, the normal load on the tires is at a maximum when the camber angle is approximately $\theta=10 \mathrm{deg}$, with the exact figure coming from the solution to Eq. (13). Given the accuracy of Eqs. (12) and (13) relative to the high-fidelity model, one would expect a similar level of veracity from Eq. (11). This is indeed the case as has been confirmed by computation; confirmatory plots have not been included in order to save space.

2.6 Tire Adhesion Limits. For a stable static equilibrium, one requires

$$
\mu_{\text {limit }}>\left|F_{y} / F_{z}\right|=\left|l_{o} \sin (\phi-\theta) /\left(l_{o} \cos (\phi-\theta)+\rho\right)\right|
$$

or what is equivalent

$$
|\phi-\theta|<\arctan \left(\mu_{\text {limit }}\right)+\arcsin \left(\frac{\mu_{\text {limit }} \rho}{l_{o} \sqrt{1+\mu_{\text {limit }}^{2}}}\right)
$$

which simplifies to

$$
|\phi-\theta|<\arctan \left(\mu_{\text {limit }}\right)
$$

in the case of the thin tire. In condition (14), $\mu_{\text {limit }}$ is the friction limit, which faces its most demanding challenge when $\phi-\theta$ is at a maximum. In the case that $\mu_{\text {limit }}=1.6, l_{o}=0.4316 \mathrm{~m}$, and $\rho$ $=0.0775 \mathrm{~m}$, the friction limit requires $|\phi-\theta|<58 \mathrm{deg}+8.8 \mathrm{deg}$ $=66.8 \mathrm{deg}$; the tire profiling increases the admissible tire-road camber angle by $8.8 \mathrm{deg}$ over that achievable with an equivalent thin tire.

If a stable equilibrium is to exist (in roll), the following condition

$$
l_{o} \sin \phi+\rho \sin \theta \geq 0
$$

must hold, which places another restriction on the admissible values of the machine roll and road camber angles. Condition (15) also shows that stable cornering can be achieved, even if the ma- chine is running with zero roll angle, providing the tire generates an overturning moment. As is clear from Fig. 3, a thick tire will generate an overturning moment by virtue of its geometry. There are two roll-related stability limits illustrated in Fig. 7 that will now be analyzed. These limits correspond to the cases in which the denominator and numerator of Eq. (11) vanish. The required positivity of the moment arms in Eq. (10) and the path radius of curvature enforce Eq. (15) as a necessary condition for a stable static roll equilibrium. In the case that the left hand side of Eq. (15) vanishes, the machine will operate in the condition illustrated in Fig. 7(a), which corresponds to straight running on a static roll stability boundary. In the case of a thin tire, which produces no overturning moment, stable equilibria can only be supported by non-negative roll angles. Since the moment arms in Eq. (11) represent non-negative distances, the following must hold;

$$
l_{o} \cos \phi+\rho \cos \theta>0
$$

In the limiting case that $v^{2} / r \rightarrow \infty$, the quantity on the left hand side of Eq. (16) tends to zero; see Eq. (11). This leads to a second roll-related stability limit that can only be approached asymptotically; a physical interpretation of this operating condition is illustrated in Fig. 7(b). The friction limits and the two roll-related stability limits are combined in Fig. 8 to define the machine's stable operating region.

The extreme "wall of death" situation is considered briefly. When $\theta=90 \mathrm{deg}$, Eq. (8) yields $F_{y}=M g$ so that

$$
F_{z} \mu_{\text {limit }}>M g
$$

is required in order to keep the machine stable. Under these conditions $F_{z}=M v^{2} / r$, so that

$$
v>\sqrt{\frac{r g}{\mu_{\text {limit }}}}
$$

is required for stable equilibrium operation. In the case that $r$ $=10 \mathrm{~m}$ and $\mu_{\text {limit }}=1.6$, the machine speed must be $v>7.83 \mathrm{~m} / \mathrm{s}$. It should be pointed out that there is nothing "special" about a camber angle of $\theta=90 \mathrm{deg}$, and other high camber angle condition can be analyzed in a straightforward way. Figure 9 illustrates the equilibrium machine roll angle as a function of $v^{2} /(\mathrm{rg})$. The curves in Fig. 9(a) come from solving Eq. (10), with the corre-

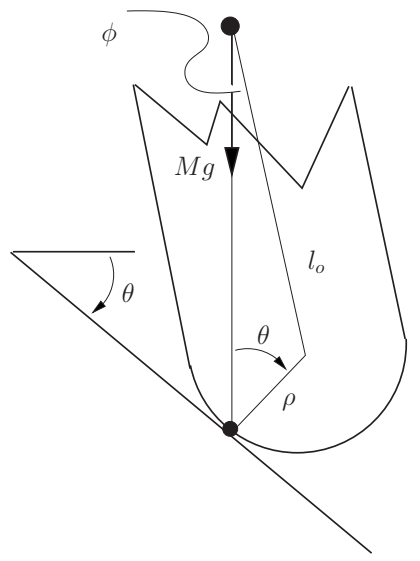

(a)

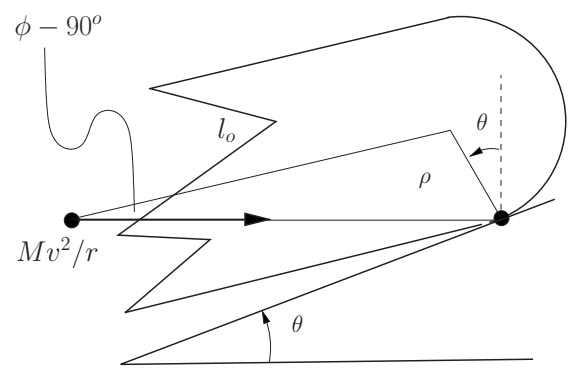

(b)

Fig. 7 Roll-angle stability limits. (a) Condition (15) shows that one static roll stability limit is reached when the machine mass center lies directly above the ground contact. It follows from Eq. (11) that under these conditions the machine's path curvature approaches zero $(r \rightarrow \infty)$ for all operating points along this stability boundary. (b) The condition $I_{0} \cos \phi+\rho \cos \theta>0$ shows that a static roll stability limit is approached when the centripetal force passes through the ground-contact point. This operating condition can only be approached asymptotically as $v^{2} / r \rightarrow \infty$; note that the gravitational force is ignored due to its negligible influence in this case. 


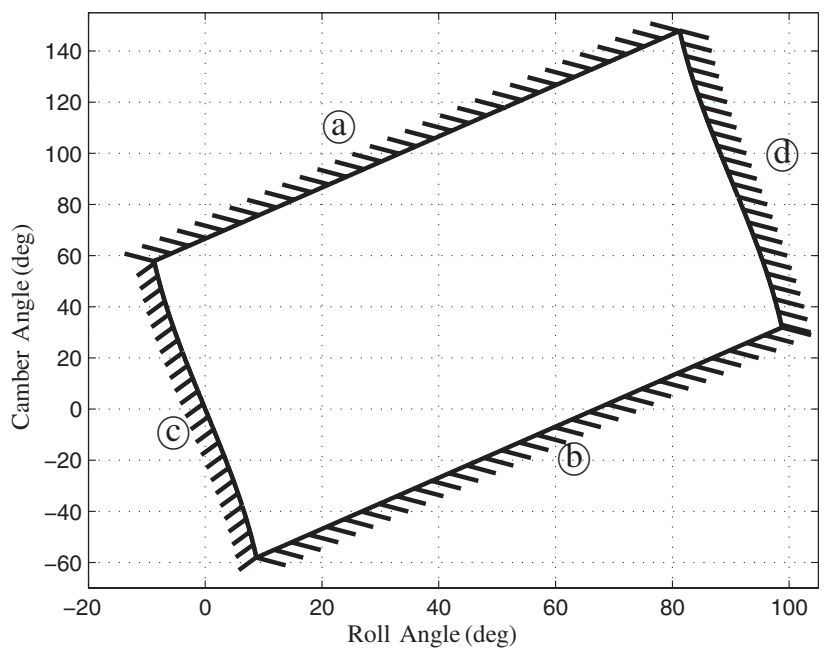

Fig. 8 Static stability limits. In order for a stable trim-state to exist, the motorcycle must operate within the cross-hatched region illustrated. This region is defined by the following: (a) is the friction limit given by $\phi-\theta>-\arcsin \left(\mu_{\text {limit }} \rho /\left(I_{o}\left(1+\mu_{\text {limit }}^{2}\right)^{1 / 2}\right)\right)$ $-\arctan \left(\mu_{\text {limit }}\right)$, in which $I_{o}=0.4316 \mathrm{~m}, \rho=0.0775 \mathrm{~m}$ and $\mu_{\text {limit }}$ $=1.6$ are used for illustration; $(b)$ is the friction limit given by $\phi$ $-\theta<\arcsin \left(\mu_{\text {limit }} \rho /\left(I_{o}\left(1+\mu_{\text {limit }}^{2}\right)^{1 / 2}\right)\right)+\arctan \left(\mu_{\text {limit }}\right)$. The limits (a) and (b) taken together come from Eq. (14). The boundary (C) is the vertical roll stability limit given by inequality $I_{o} \sin \phi$ $+\rho \sin \theta \geqslant 0$, (see Fig. 7(a)); (d) horizontal roll stability limit given by inequality $I_{o} \cos \phi+\rho \cos \theta>0$ (see Fig. $7(b)$ ). Under wall of death conditions, the road camber angle is given by $\theta=90 \mathrm{deg}$, and stable roll angles exist between $\phi=23.5 \mathrm{deg}$ and roll angles approaching $90 \mathrm{deg}$.

sponding machine operation condition illustrated in Fig. 9(b), where the resultant of the gravitational and centripetal forces pass through the tire-road contact point. Observe how higher values of road camber angles require higher minimum values of $v^{2} /(\mathrm{rg})$ and lower machine roll angles that approach the boundary (d) in Fig. 8 asymptotically.

\section{Results}

The main results of this paper are now presented and comprise root-locus diagrams, or plots derived from eigenvalue calculations, which were generated from a symbolic linearization of the high-fidelity model described in Sec. 2. All the results are for the open-loop machine, and so the drive and roll controllers are removed from the linearized model used to generate the results presented here. The computation of the machine's eigenvalues is a two-step process in which the trim states are found prior to the evaluation of the linearized model and the eigenvalue calculations. In the first stage of the process, the controllers described in Secs. 2.1 and 2.3 are used to drive the nonlinear model into a desired trim condition. Once the desired trim condition has been established, the trim states are used evaluate the state-space A matrices of the linearized models.

The first result, shown in Fig. 10, is a set of root-locus diagrams for the machine operating at zero roll angle. The speed is increased from $5 \mathrm{~m} / \mathrm{s}$ to $75 \mathrm{~m} / \mathrm{s}$, with four road camber angles considered. Since the vehicle roll angle is $\phi=0 \mathrm{deg}$, it follows from inequality (15) that only positive road camber angles can be considered. The zero road camber (flat road) curve is a familiar plot that has been referred to in previous papers $[8,9,36]$. It can be seen from Fig. 10 that the wobble-mode frequency varies between approximately $47 \mathrm{rad} / \mathrm{s}$ and $57 \mathrm{rad} / \mathrm{s}$, while the weave mode resonant frequency varies between $9 \mathrm{rad} / \mathrm{s}$ and $28 \mathrm{rad} / \mathrm{s}$. Apart from relatively low speeds (below $15 \mathrm{~m} / \mathrm{s}$ ), the damping of the wobble and weave modes decreases with increased speed. The key observation from Fig. 10 is the fact that the damping of both the wobble and weave modes decreases with increased road camber for speeds below $30 \mathrm{~m} / \mathrm{s}$, while at high speeds, this trend reverses and both the wobble- and weave-mode dampings increase with increased road camber. In order to clarify the quantitative nature of these trends, the real parts of the wobble- and weave-mode eigenvalues, as a function of speed, are plotted in Fig. 11. Figure

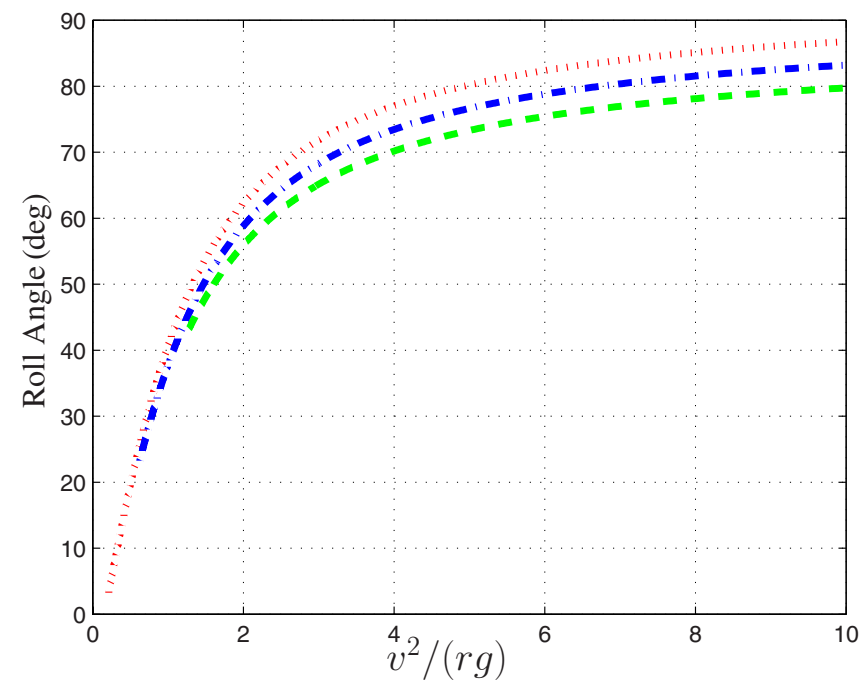

(a)

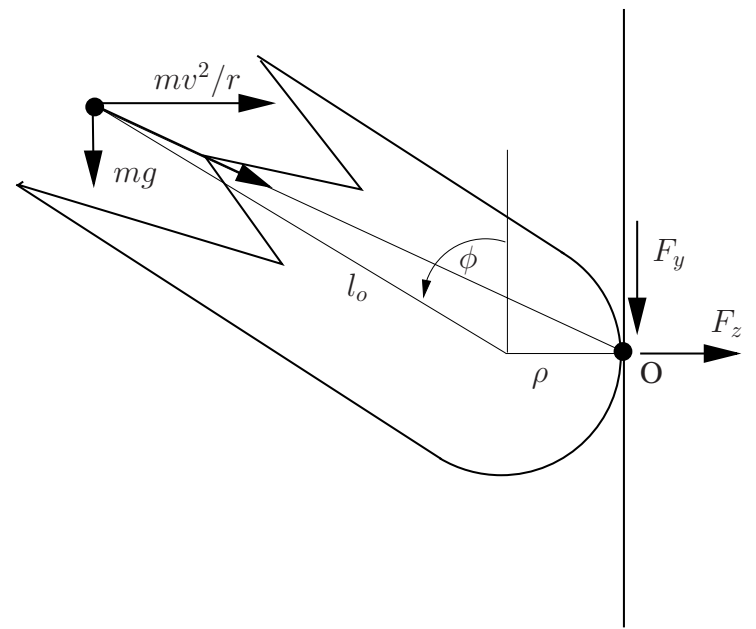

(b)

Fig. 9 Stable operation under wall of death type conditions. (a) shows the equilibrium roll angle as a function of $v^{2} /(r g)$, and was generated using Eq. (10) for three different road camber angles, and with $I_{o}=0.4316 \mathrm{~m}$ and $\rho=0.0775 \mathrm{~m}$. The dotted curve corresponds to a camber angle of $\theta=70 \mathrm{deg}$, the dot-dash curve to $\theta=90 \mathrm{deg}$, and the dashed curve to $\theta=110$ deg. For $\theta$ $>90 \mathrm{deg}$, the vehicle traverses the interior of a noninverted cone; the lowest viable value of $v^{2} /(\mathrm{rg})$ comes from the friction limit condition $\phi-\theta>-\arcsin \left(\mu_{\text {limit }} \rho /\left(I_{o}\left(1+\mu_{\text {limit }}^{2}\right)^{1 / 2}\right)\right)-\arctan \left(\mu_{\text {limit }}\right)$ (boundary (a) in Fig. 8), while the limiting value associated with $v^{2} /(r g) \rightarrow \infty$ and the corresponding highest roll angles come from the horizontal roll stability limit (boundary @d). (b) shows the motorcycle in roll equilibrium against the wall of death; the resultant of the gravitational and centripetal forces act through the tire contact point $O$. 


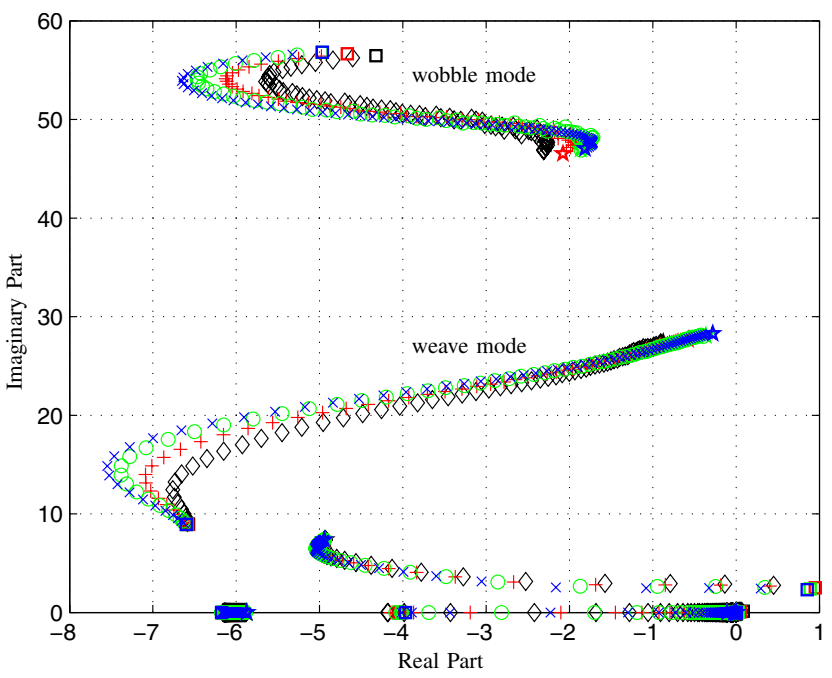

Fig. 10 Root loci for four road camber angles showing the wobble- and weave-mode eigenvalues as a function of speed. The motorcycle roll angle is $\phi=0 \mathrm{deg}$, and the speed is varied from $5 \mathrm{~m} / \mathrm{s}$ to $75 \mathrm{~m} / \mathrm{s}$. The highest speed is marked with a $\star$ and the lowest speed with a $\square$. The road cambers are annotated as 0 deg, $\times ; 5$ deg, $\bigcirc ; 10$ deg, +; 15 deg, $\diamond$.

11(a) shows that at low speed a road camber angle of $15 \mathrm{deg}$ increases the real part of the wobble-mode eigenvalue from approximately -5.0 to -4.3 ; at $15 \mathrm{~m} / \mathrm{s}$, it increases from -6.7 to -5.7 ; at $30 \mathrm{~m} / \mathrm{s}$, there is virtually no change, while at $75 \mathrm{~m} / \mathrm{s}$, the trend reverses and the real part of the wobble mode decreases from -1.8 to -2.3 as road camber is introduced. The high-speed trend is the most important, because at high speed the wobblemode damping is at its lowest, and the introduction of $15 \mathrm{deg}$ of road camber causes the wobble-mode half-amplitude decay time to increase by almost $25 \%$ from $0.3 \mathrm{~s}$ to $0.38 \mathrm{~s}$. Figure $11(b)$ shows that at low speed a road camber increase of $15 \mathrm{deg}$ has little or no effect on the weave-mode damping; at $15 \mathrm{~m} / \mathrm{s}$, the real part of the weave-mode eigenvalue increases from -7.6 to -6.5 ; there is no change in the weave-mode damping at $30 \mathrm{~m} / \mathrm{s}$, while at $75 \mathrm{~m} / \mathrm{s}$, the real part of the weave-mode eigenvalue decreases from -0.3 to -0.9 . Again, the high-speed trend is the most important one from a safety perspective, because under these conditions the weave mode is lightly damped and the introduction of road camber causes the half-amplitude decay time to decrease from $2.3 \mathrm{~s}$ to $0.77 \mathrm{~s}$. The overall conclusion is that the damping of the high-speed wobble and weave modes both decrease as the vehicle becomes more nearly perpendicular to the road surface.

Figure 12 shows a set of root-locus diagrams for the machine operating at $5 \mathrm{deg}$ and $15 \mathrm{deg}$ of roll angle. Direct calculation using Eq. (15) shows that at 5 deg of roll angle, the road camber can be decreased to -29 deg without a loss of static roll stability. Therefore, in comparison to Fig. 10, the range of road camber angles investigated can be expanded to include adverse (negative) road camber conditions. The key observation from Fig. 12(a) is the fact that the high-speed damping of both the wobble and weave modes is lowest at $5 \mathrm{deg}$ of road camber, which is the condition under which the tires are almost perpendicular to the road. The damping of both modes then gets progressively higher as the angle between the tires and the road increases. It will be shown in a subsequent figure that this trend is insensitive to the sign of the tire-to-road roll-angle increase. As before, at low speed, both the wobble- and weave-mode dampings are greatest when the road camber angle is $5 \mathrm{deg}$. Figure $12(\mathrm{~b})$ serves only to reinforce the observation that the high-speed weave- and wobblemode dampings are lowest when the road tires are perpendicular to the road and that this trend then reverses at low speed. In order to clarify the quantitative nature of these trends, the real parts of the wobble and weave modes, as a function of road camber angle, are plotted in Figs. 13 and 14, respectively. In each case, the $(a)$ plot corresponds to $10 \mathrm{~m} / \mathrm{s}$, which is representative of the lowspeed behavior, while the $(b)$ plots correspond to the $75 \mathrm{~m} / \mathrm{s}$, which is representative of high speeds. At low speeds, it can be seen that the wobble-mode damping is greatest when the road camber angle and the machine roll angle are equal, or in other words when the tire is perpendicular to the road. This is true of each of the four machine-roll-angle test conditions. It is also interesting to note that the damping of both modes is insensitive to the sign of the tire-road camber angle. For the 5 deg roll-angle case, the modal damping of both modes are very similar for road camber angles of zero and $10 \mathrm{deg},|\phi-\theta|=5 \mathrm{deg}$ and is the same in each case. Indeed, these plots indicate a direct relationship between the modal damping and the tires' normal load, which is a function of $|\phi-\theta|$ rather than $\phi-\theta$; note that $F_{z}$ is an even function of $\phi-\theta$; see Eq. (13). At high speed, the opposite is true in that an orthogonal relationship between the tire and the road is detrimental to the stability of the high-speed wobble mode. In the

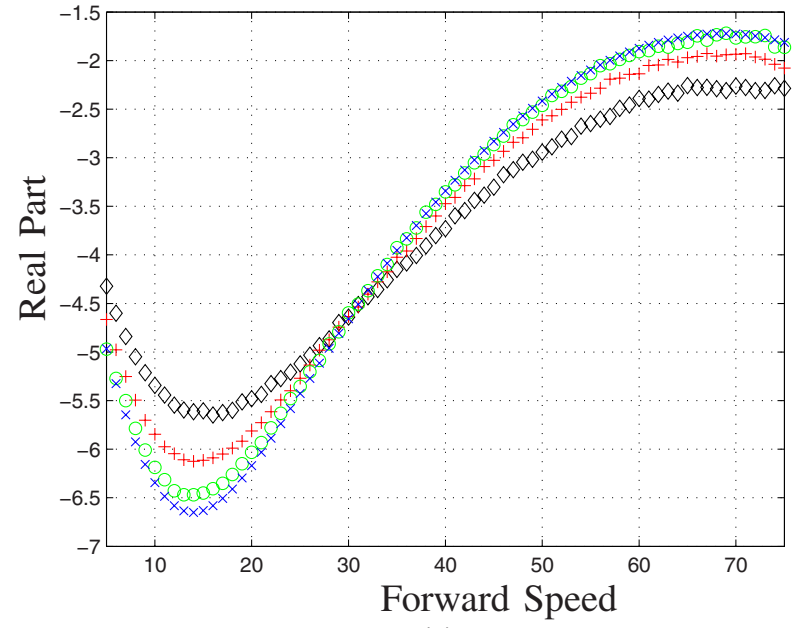

(a)

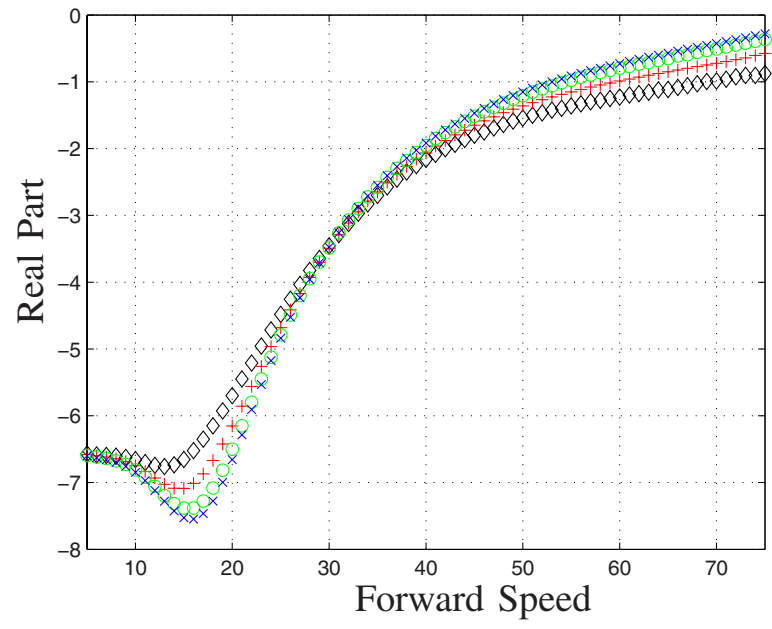

(b)

Fig. 11 Real parts of the wobble- and weave-mode eigenvalues as a function of speed for four different road camber angles, and a motorcycle roll angle of $\phi=0 \mathrm{deg}$. The real part of the wobble-mode eigenvalue is shown in (a), while the real part of the weave-mode eigenvalue is shown in $(b)$. The road camber curves are annotated as 0 deg, $\times ; 5$ deg, $\bigcirc ; 10$ deg, +; 15 deg, $\diamond$. 


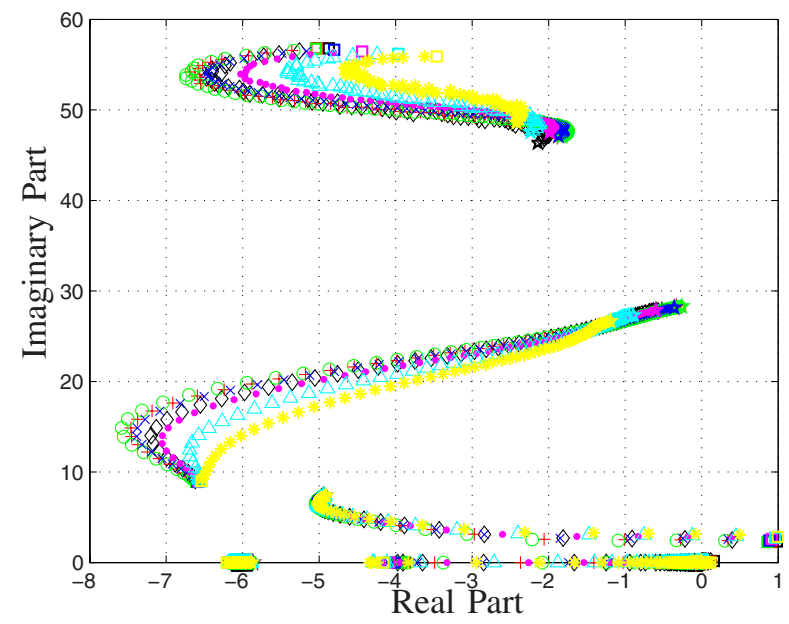

(a)

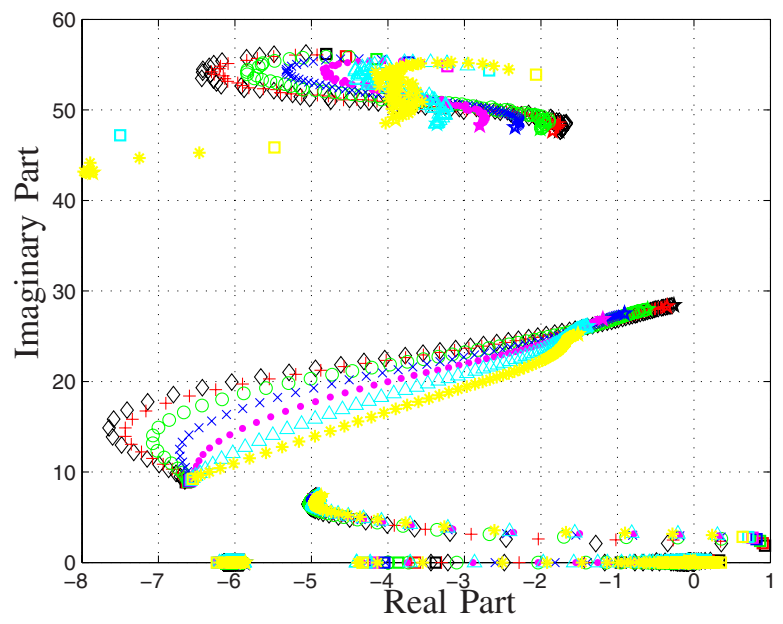

(b)

Fig. 12 Root loci for seven road camber angles, with a motorcycle roll angle of $\phi=5$ deg (a), and $\phi=15$ deg $(b)$. The speed is varied from $5 \mathrm{~m} / \mathrm{s}$ to $75 \mathrm{~m} / \mathrm{s}$; the highest speed is marked with $a \star$ and the lowest speed with a $\square$. The road cambers are annotated as $-15 \mathrm{deg}, * ;-10 \mathrm{deg}, \triangle ;-5 \mathrm{deg}, 0 ; 0 \mathrm{deg}, \times ; 5 \mathrm{deg}, \bigcirc ; 10 \mathrm{deg},+; 15 \mathrm{deg}, \diamond$.

extreme high-speed case corresponding to a machine roll angle of $15 \mathrm{deg}$, the wobble-mode half-amplitude decay time increases from $0.1507 \mathrm{~s}(\theta=-20 \mathrm{deg})$ to $0.41 \mathrm{~s}(\theta=20 \mathrm{deg})$.

The road camber-related behavior of the weave mode is examined in detail in Fig. 14. As with the wobble mode, at low speeds, it can be seen that the weave-mode damping is greatest when the road camber angle and the machine roll angle are equal, or when the tire is perpendicular to the road. In the extreme case, at low speed, which corresponds to a machine roll angle of $15 \mathrm{deg}$, the real part of the weave-mode eigenvalue increases from -6.8 at a positive road camber angle of $\theta=20 \mathrm{deg}$ to -5.7 at an adverse camber of $\theta=-20 \mathrm{deg}$. The important case corresponds to the $75 \mathrm{~m} / \mathrm{s}$ high-speed situation when positive camber has a destabilizing influence on the weave mode. As with the wobble mode, at high speed, an orthogonal relationship between the tire and the road is destabilizing. In the extreme high-speed case corresponding to a machine roll angle of $15 \mathrm{deg}$, the weave-mode half-amplitude decay time increases from $0.46 \mathrm{~s} \quad(\theta$ $=-15 \mathrm{deg})$ to $2.48 \mathrm{~s}(\theta=15 \mathrm{deg})$.

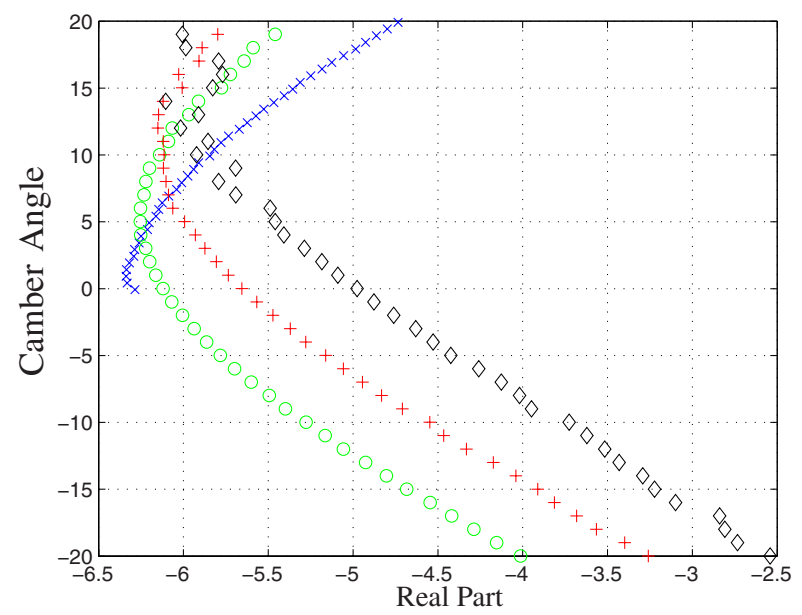

(a)
The concluding set of results is for a moderately high roll angle of $\phi=30 \mathrm{deg}$. The central aim is to establish that the trends alluded to above hold good at higher vehicle roll angles. Figure 15 shows a set of root loci corresponding to a machine roll angle of $30 \mathrm{deg}$; the speed is increased from $5 \mathrm{~m} / \mathrm{s}$ to $75 \mathrm{~m} / \mathrm{s}$, with seven road camber angles considered. It is clear that the high-speed weave-mode behavior retains its prior behavior with its damping increasing as the tire-to-road camber angle increases. It is also clear that the low-speed weave-mode damping is relatively insensitive to road camber. It is not clear from this plot if there is a low-speed trend reversal of the type observed earlier. The computation of the wobble-mode behavior at high roll angles proved problematic, because despite extensive controller tuning, it is difficult to maintain a constant trim state under these operating conditions. The overall trends are still clear. At high speeds, the wobble-mode damping decreases as the tire becomes more nearly orthogonal to the road, with this trend reversing at lower speeds. Quantitative clarity is established in Fig. 16 where the real parts of

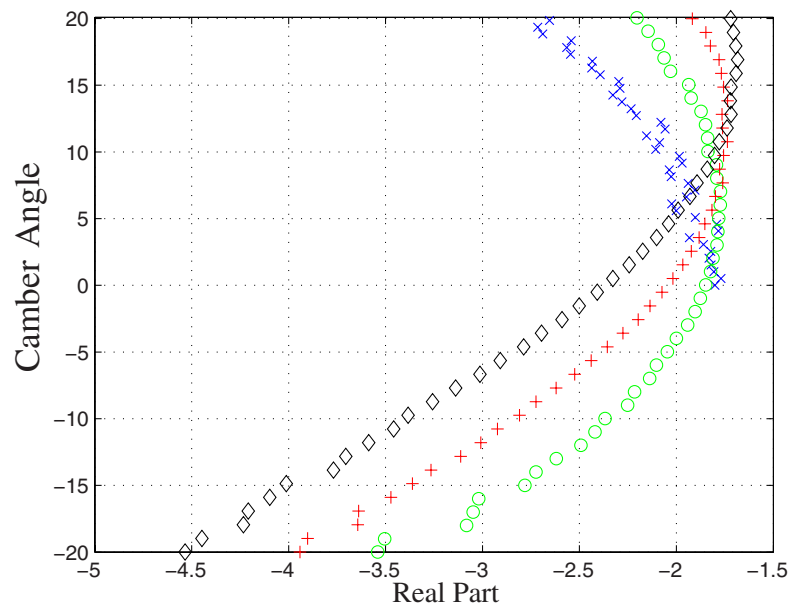

(b)

Fig. 13 Real part of the wobble-mode eigenvalue as a function of the road camber angle for four different machine roll angles. The motorcycle's forward speed is constant at (a) $10 \mathrm{~m} / \mathrm{s}$ and (b) $75 \mathrm{~m} / \mathrm{s}$. The motorcycle roll-angle curves are annotated as 0 deg, $\times$; 5 deg, $\bigcirc ; 10$ deg, +; 15 deg, $\diamond$. 


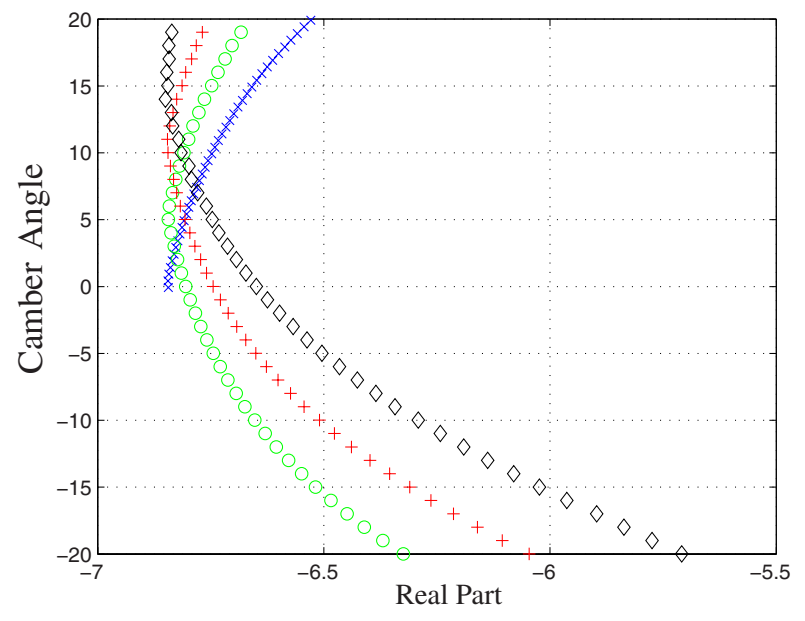

(a)

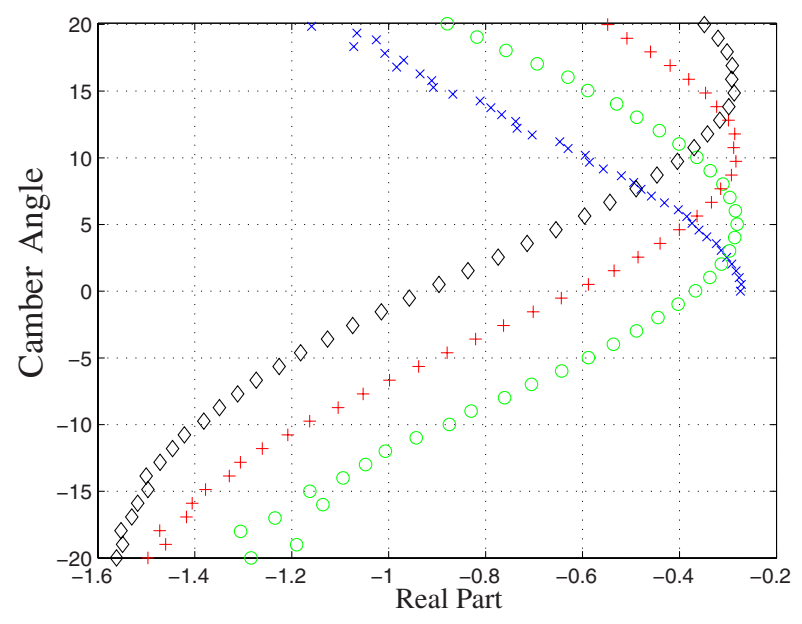

(b)

Fig. 14 Real part of the weave-mode eigenvalue as a function of the road camber angle for four different machine roll angles. The motorcycle's forward speed is constant at (a) $10 \mathrm{~m} / \mathrm{s}$ and (b) $75 \mathrm{~m} / \mathrm{s}$. The motorcycle roll-angle curves are annotated as 0 deg, $\times ; 5$ deg, $\bigcirc ; 10$ deg, +; 15 deg, $\diamond$.

the wobble- and weave-mode eigenvalues are plotted as a function of speed for seven different road camber angles, and a motorcycle roll angle of $\phi=30 \mathrm{deg}$. The $(a)$ plot shows that the low-speed wobble mode is most damped for high positive camber and least damped for high negative camber, with the 30 deg change in camber angle decreasing the real part of the wobble mode from approximately -1.0 to -3.4 . The wobble-mode damping then becomes relatively insensitive to camber at $40 \mathrm{~m} / \mathrm{s}$, with the wobble-mode damping trend again reversing at high speed. The (b) plot shows that the low-speed weave-mode damping is insensitive to camber, with the real part of the weave mode increasing from -6.7 to -6.3 as the road camber is swept from $15 \mathrm{deg}$ to $-15 \mathrm{deg}$. This trend, such as it is, reverses at a speed of approximately $50 \mathrm{~m} / \mathrm{s}$. At $75 \mathrm{~m} / \mathrm{s}$, the weave-mode damping is lowest for high positive camber and highest for maximum adverse camber, with the change in the real part of the weave-mode eigenvalue similar to that observed in the zero roll-angle case.

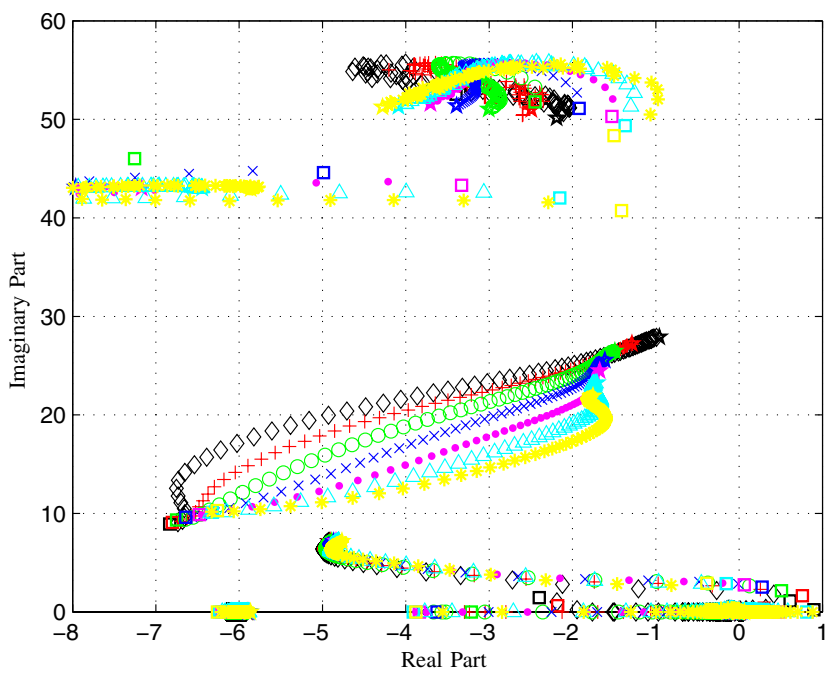

Fig. 15 Root-loci for seven road camber angles, with a motorcycle roll angle of $\phi=30 \mathrm{deg}$; the speed is varied from $5 \mathrm{~m} / \mathrm{s}$ to $75 \mathrm{~m} / \mathrm{s}$. The highest speed is marked with $a \star$ and the lowest speed with a $\square$. The road cambers are annotated as $-15 \mathrm{deg}$, *; $-10 \mathrm{deg}, \triangle ;-5 \mathrm{deg}, 0 ; 0 \mathrm{deg}, \times ; 5 \mathrm{deg}, \bigcirc ; 10 \mathrm{deg}$, $+; 15 \mathrm{deg}, \diamond$.

\section{Conclusions}

The influence of road camber on motorcycle stability is an issue that sometimes arises in loss-of-control motorcycle accident investigations. A typical scenario involves high-speed cornering on a superelevated motorway. The overarching contribution of this paper is to clarify the influence of road camber on the stability of the two important oscillatory modes of behavior associated with single-track vehicles known as wobble and weave. It must be emphasized that the conclusions drawn pertain to a particular class of high-powered sports motorcycles, and are not claimed to be "true for all motorcycles."

Contributions include a method of "forcing" a complex nonlinear simulation model to operate stably at a prescribed fixed speed, fixed roll-angle, and fixed road-camber-angle operating condition. This involved a number of enhancements to an existing model including the introduction of local tangent plane approximations for a conical road surface. Separate tangent planes are required for each of the road tires, since extensive force, moment, and power balance testing established that one such tangent plane was inadequate at low speeds. It was also found necessary to introduce adaptive references into both the roll-angle steering control loop and the rear-wheel drive loop. The first of these adaptive references was used to center the machine's cornering trajectory on an arbitrary prescribed point in the ground plane (usually the origin $n_{0}$ of the inertial reference frame). Adaptation in the speed reference of the rear-wheel drive controller was used to remove persistent low-frequency height oscillations that can occur as the vehicle revolves around its cornering trajectory. This phenomenon is at its most troublesome at large radii of cornering curvature.

Important insights are obtained from the simple static force and moment balance calculations presented in Secs. 2.5 and 2.6. It is believed that the single-wide-tire model presented represents a good balance between fidelity and simplicity for the purposes to which it is put here. These include the analysis of the way in which the steady-state tire normal-load and side forces vary with road camber, the calculation of the machine's cornering radius of curvature as a function of speed, roll angle and road camber angle, and the quantitative characterization of the static roll stability boundary. The veracity of this simple analysis was established by comparison to the complex dynamic model presented.

The conventional wisdom as it is taught to advanced police riders [1], which is based on simple equilibrium considerations, focuses on achieving riding conditions that minimize steady-state tire side forces and maximize the tire normal loading. While this 


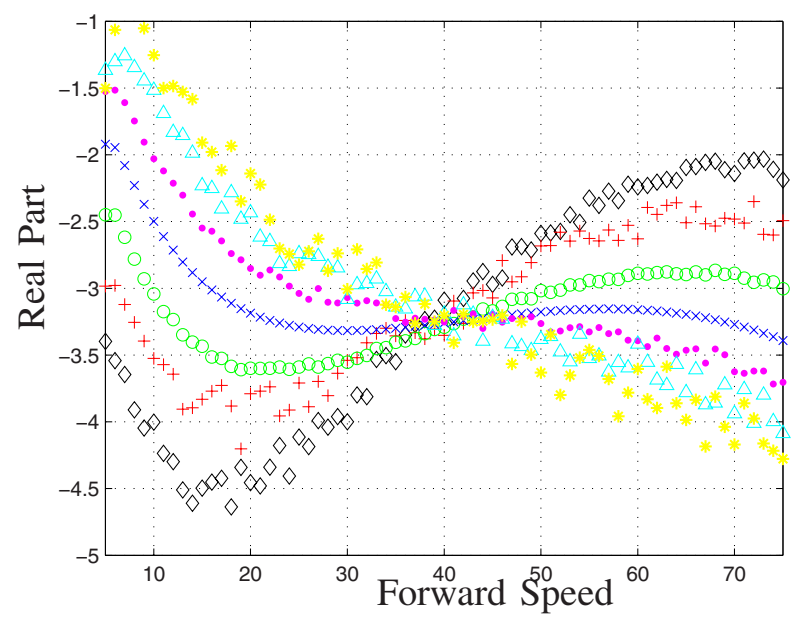

(a)

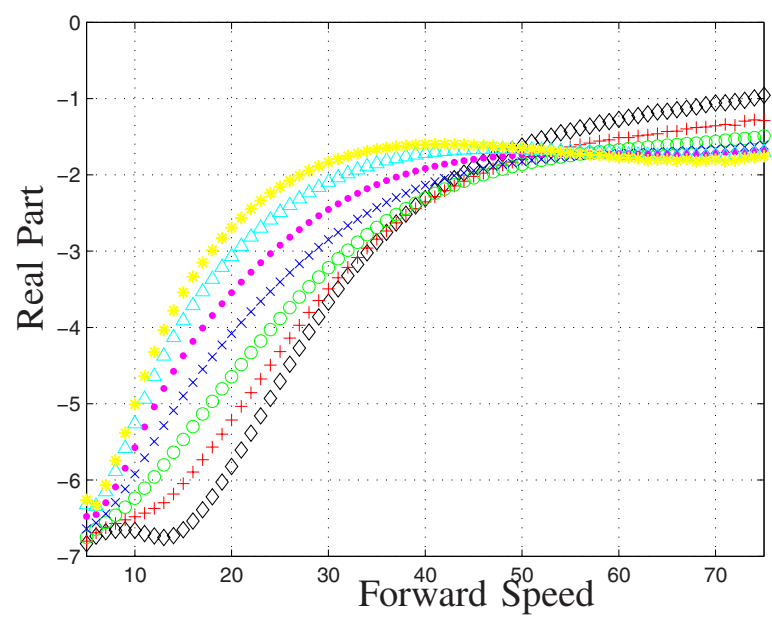

(b)

Fig. 16 Real parts of the wobble- and weave-mode eigenvalues as a function of speed for seven different road camber angles and a motorcycle roll angle of $\phi=30 \mathrm{deg}$. The real part of the wobble-mode eigenvalue is shown in the (a) figure, while the weave-mode behavior is shown in the (b) plot. The road camber curves are annotated as $\mathbf{- 1 5}$ deg, $* ;-10$ deg, $\triangle ;-5$ deg, 0 ; 0 deg, $\times ; 5$ deg, $\bigcirc ; 10$ deg, +; 15 deg, $\diamond$.

is probably the best thing to do in the context of four-wheeled vehicles, it is not necessarily the right advice to give to motorcyclists. It has been demonstrated by high-fidelity dynamic modeling that the damping of the high-speed wobble and the high-speed weave modes are at their minimum when the road tires are vertical to the road. As the tire-road inclination angle increases, the high-speed wobble and weave-mode damping increases also. This increased damping does not appear sensitive to the sign of the tire-to-road inclination angle $(\theta-\phi)$ and appears instead sensitive to $|\theta-\phi|$; see Figs. 13 and 14. It is therefore concluded that increased tire loading has a destabilizing effect on the high-speed wobble- and high-speed weave-mode stabilities, because it too increases with $|\theta-\phi|$; see Eq. (13) and the simplification that appears immediately after it. At low speeds, these trends reverse and the wobble and weave-mode dampings increase as the tires become more nearly vertical with the road. Under these more benign conditions, the conventional police training advice appears to hold good.

\section{Acknowledgment}

This work was supported by a Portfolio Partnership held with the British Engineering and Physical Sciences Research Council.

\section{References}

[1] Coyne, P., 1996, Motorcycle Roadcraft: The Police Rider's Handbook, Stationary Office Books, London.

[2] Limebeer, D. J. N., and Sharp, R. S., 2006, "Bicycles, Motorcycles and Models," IEEE Control Syst. Mag., 26(50), pp. 34-61.

[3] Meijaard, J. P., Papadopoulos, J. M., Ruina, A., and Schwab, A. L., 2007, "Linearized Dynamics Equations for the Balance and Steer of a Bicycle: A Benchmark and Review," Proc. R. Soc. London, Ser. A, 463, pp. 1955-1982.

[4] Koenen, C., 1983, "The Dynamic Behaviour of Motorcycles When Running Straight Ahead and When Cornering," Ph.D. thesis, Delft University of Technology, Delft, Netherlands.

[5] Sharp, R. S., and Limebeer, D. J. N., 2001, “A Motorcycle Model for Stability and Control Analysis,” Multibody Syst. Dyn., 6(2), pp. 123-142.

6] Cossalter, V., and Lot, R., 2002, "A Motorcycle Multi-Body Model for Real Time Simulations Based on the Natural Coordinates Approach," Veh. Syst. Dyn., 37(6), pp. 423-447.

[7] Sharp, R. S., 2001, "Stability, Control, and Steering Responses of Motorcycles," Veh. Syst. Dyn., 35(4,5), pp. 291-318.

[8] Sharp, R. S., Evangelou, S., and Limebeer, D. J. N., 2003, "Improved Modelling of Motorcycle Dynamics," ECCOMAS Thematic Conference on Advances in Computational Multibody Dynamics, J. Ambósio, ed., Lisbon, Jul. 1-4.

[9] Sharp, R. S., Evangelou, S., and Limebeer, D. J. N., 2004, "Advances in the Modelling of Motorcycle Dynamics,” Multibody Syst. Dyn., 12(3), pp. 251281.

[10] Döhring, E., 1956, "Steering Wobble in Single-Track Vehicles," ATZ, 58(10), pp. 282-286.

[11] Eaton, D. J., 1973, "Man-Machine Dynamics in the Stabilization of SingleTrack Vehicles," Ph.D., thesis, University of Michigan.

[12] Watanabe, Y., and Yoshida, K., 1973, "Motorcycle Handling Performance for Obstacle Avoidance," Second International Congress on Automotive Safety, San Francisco.

[13] Jennings, G., 1974, "A Study of Motorcycle Suspension Damping Characteristics," SAE Paper No. 740628

[14] Roe, G. E., and Thorpe, T. E., 1976, "A Solution of the Low-Speed Wheel Flutter Instability in Motorcycles,” J. Mech. Eng. Sci., 18(2), pp. 57-65.

[15] Verma, M. K., 1978, "Theoretical and Experimental Investigation of Motorcycle Dynamics," Ph.D. thesis, University of Michigan.

[16] McKibben, J. S., 1978, "Motorcycle Dynamics-Fact, Fiction and Folklore," SAE Paper No. 780309

[17] Aoki, A., 1979, "Experimental Study on Motorcycle Steering Performance," SAE Paper No. 790265

[18] Weir, D. H., and Zellner, J. W., 1979, "Experimental Investigation of the Transient Behaviour of Motorcycles," SAE Paper No. 790266.

[19] Thomson, B., and Rathgeber, H., 1984, "Automated Systems Used for Rapid and Flexible Generation of System Models Exemplified by a Verified Passenger Car and a Motorcycle Model," Dynamics of Vehicles on Roads and on Railway Tracks, J. K. Hedrick, ed., Swets and Zeitlinger, Lisse, pp. 645-654.

[20] Bayer, B., 1988, "Flattern und pendeln bei krafträdern," Automobil Industrie, 2, pp. 193-197.

[21] Takahashi, T., Yamada, T., and Nakamura, T., 1984, "Experimental and Theoretical Study of the Influence of Tires on Straight-Running Motorcycle Weave Response," SAE Paper No. 840248.

[22] Otto, W. M., 1980, "Effect of Motorcycle Accessories on Stability," Proceedings of International Motorcycle Safety Conference, Linthicum, MD, Motorcycle Safety Foundation, Washington, pp. 1560-1581.

[23] Sugizaki, M., and Hasegawa, A., 1988, "Experimental Analysis of Transient Response of Motorcycle Rider Systems," SAE Paper No. 881783.

[24] Biral, F., Bortoluzzi, D., Cossalter, V., and Da Lio, M., 2003, "Experimental Study of Motorcycle Transfer Functions for Evaluating Handling," Veh. Syst. Dyn., 39(1), pp. 1-26.

[25] Evangelou, S., and Limebeer, D., 2000, "LISP Programming of the "Sharp 1971" Motorcycle Model," http://www.imperial.ac.uk/controlandpower/ motorcycles/

[26] Sharp, R. S., and Alstead, C. J., 1980, "The Influence of Structural Flexibilities on the Straight Running Stability of Motorcycles," Veh. Syst. Dyn., 9(6), pp. 327-357.

[27] Spierings, P. T. J., 1981, "The Effects of Lateral Front Fork Flexibility on the Vibrational Modes of Straight-Running Single-Track Vehicles," Veh. Syst. Dyn., 10(1), pp. 21-35.

[28] Limebeer, D. J. N., Sharp, R. S., and Evangelou, S., 2002, "Motorcycle Steering Oscillations Due to Road Profiling," ASME J. Appl. Mech., 69(6), pp. 724-739.

[29] de Vries, E. J. H., and Pacejka, H. B., 1998, "The Effect of Tyre Modeling on the Stability Analysis of a Motorcycle," Proceedings of AVEC'98, Nagoya, Japan, pp. 355-360.

[30] de Vries, E., and Pacejka, H., 1998, "Motorcycle Tyre Measurements and Models," Proceedings of 15th IAVSD Symposium on the Dynamics of Vehicles on Roads and on Tracks, L. Palkovics, ed., Budapest, Hungary, Aug. 25-29, 1997, Veh. Syst. Dyn. 28, pp. 280-298. 
[31] Pacejka, H. B., 2002, Tyre and Vehicle Dynamics, Butterworth-Heinemann, Oxford.

[32] Tezuka, Y., Ishii, H., and Kiyota, S., 2001, “Application of the Magic Formula Tire Model To Motorcycle Maneuverability Analysis," JSAE Rev., 22, pp. 305-310.

[33] Mechanical Simulation Corporation, 1998, AUTOSIM 2.5+ Reference Manual, Ann Arbor, MI, http://www.carsim.com
[34] Kreyszig, E., 1967, Advanced Engineering Mathematics, Wiley, New York.

[35] Timoshenko, S., and Young, D. H., 1948, Advanced Dynamics, McGraw-Hill, New York.

[36] Evangelou, S., Limebeer, D. J. N., Sharp, R. S., and Smith, M. C., 1967, "Mechanical Steering Compensators for High-Performance Motorcycles," ASME J. Appl. Mech., 74, pp. 332-346. 